262 Franz Friedmann: Rudimentäre Eier im Hoden etc.

\title{
Erklärung der Abbildungen auf Tafel XV.
}

Sämmtliche Abbildungen sind in ihren Contouren mit Hülfe des Abbéschen Zeichenapparates in der Höhe des Objekttisches entworfen. Fig. 1. ist mit Zeiss Objektiv AA und Ocular 4 gezeichnet.

Fig. 2 a, b, c, d ist mit Himmler homogen. Immers. $1 / 12$ und Ocular Zeiss 2 gezeichnet.

Fig. 3. ist mit Zeiss Objektiv DD und Ocular 2 gezeichnet.

Fig. 4. ist mit Zeiss Objektiv DD und Ocular 2 gezeichnet.

Siammtliche Figuren stellen mit Hermann'scher Flüssig'keit fixirte Präparate dar, Fig. 1-3 mit Färbung in Saffranin und Gentianaviolett, Fig. 4 ohne weitere Farbung.

Fig. 1. Schnitt durch einen Tubul. semin., der durch das in ihm liegende Ei grossentheils ausgefült wird und nur noch einen schmalen Halbmond von Hodengewebe zeigt.

Fig. $2 a, b, c$ und d. Segmente von Keimbläschen, in denen die Chromatinknaiuel liegen. Siimmtliche 4 Zeichnungen stammen von verschiedenen Eirun.

Fig. 3, Ein Hodenei im Stadium der Degeneration.

Fig. 4. Schnitt durch einen Tubulus seminiferus mit reger Spermatogenese. Derselbe enthialt in seinern Lumen ein kleines Fi in Stadiuın vorgeschrittener Degeneration.

\section{Neues über petrificirte Muskulatur etc.}

Von

Dr. Otto M. Reis in München.

In Bd. XXXXI und XXXXIV dieser Zeitschrift hat der Verfasser mikroskopische Untersuchungen über eine PhosphoritPetrificirung der Muskulatur, des Nervensystems und der Lederliaut fossiler Würmer, Cephalopoden, Fische und Reptilien veröffentlicht, welche in zoologischen Kreisen ein viel grösseres Interesse erregten und besseres Verständniss fanden, als in paläontologischen; es sei daher grestattet, einiges Nene hier noch kurz 
zu berühren, besonders auch einige Besprechungen der Petrificirungserscheinung in der neuesten paläontologischen Litteratur zu prüfen.

Abgesehen von einer kurzen Berührung der schon $\mathrm{N}$ ew berr $\mathrm{y}$ 1389 bekannten und von ihm abgebildeten (vgl. S. 264 Anm.) Thatsache durch 0 . Jaekel bei dem in Nordamerika gefundenen palaeozoischen Elasmobranchier CI ad od us (Cladosela che) in Sitzber. der Ges. natf. Freunde zu Berlin 1892 (S. 80-92), hat besonders v. Ammon (vergl. Berichte des naturw. Vereins zu Regensburg: 1894/95, V. Heft) die von mir bei Ischyodus dargestellten Einzelheiten, soweit sie makroskopisch zu beobachten sind, alle an einem nenen wohlerhaltenen Exemplar wiedererkannt.

Was nun meine Beobachtungen über Petrificirung der Muskulatur bei Reptilien betrifft, so ist neuerdings W. D a mes gelegentlich eines "Beitrag's zur Kenntniss der Gattung $\mathrm{Pl}$ e u r $0^{-}$ s a urus (H. v. Meyer)" mit der Annahme einer neuen Entdeckung (cf. Sitzber. d. k. preuss. Akademie der Wissenschaften zu Berlin, 1896, S. 1109) aufgetreten. Er bespricht daselbst Abdrücke von Weichtheilen, welche ungefähr der Länge der einzelnen Wirbel entsprechen und bemerkt: "solche Eindrücke der Weichtheile sind meines Wissens an fossilen Wirbelthieren noch nicht beobachtet worden und ich bin Herrn $D$ u B o is-Reymond zu lebhaftem Dank verpflichtet, dass er mich bei Besichtigung derselben sofort auf Myocommata hinwies." In einer Anmerkung: nıacht Dames nur auf die Erwähnung der „Ligamentinscriptionen del Muskelmassen (Myocommata)" bei Ischyodus in der oben erwähnten Schrift v. A m mon's aufmerksam, deren genauere Beschreibung und Abbildung für später in Aussicht gestellt sei!

Es muss hierbei Wunder nehmen, dass Dames mit keinem Wort auf das von mir verschiedentlich erwähnte Auftreten der Myocommata (Ligamentinscriptionen der Rumpfmuskulatur) zurückkommt und etwaige Beziehungen oder Verschiedenheiten im Vergleich mit der von ihm beobachteten Erscheinung aufdeckt. Ich habe "Ligamentinscriptionen" nicht nur bei Undina (Paläontogr. Bd. XXXV, Taf. 2, Fig. 5) in allergrösster Deutlichkeit abgebildet, bin in meiner I. Detailabhandlung ausführlich darauf zurückgekommen (S. 493 und 513), habe auch daselbst Taf. XXX Theile der Myocommata von Call opterus abgebildet und von Is chyodus 
eriualint, sondern habe auch auf Abbildungen von A canthodes nach Kner, Chenacanthus nach Traquair, Agassizia nach Vette r (bzw. S. 508, 509 und 519) verwiesen, wo eben die Myocommata in mehr weniger klarer Ausbildung die makroskopische Grundlage vermuthungsweiser Deutung ,versteinerten Fleisches“ bildeten ${ }^{1}$ ). Ausserdem sind diese Punkte in einem sachlichen Musterreferat von Dr. $R$ a $\mathrm{uff}$ in dem von $\mathrm{D}$ a mes redigirten Neuen Jahrbuch für Mineralogie 1895 ausführlichst erwähnt.

Wir sind also gezwungen, auf eigne Hand den Vergleich zu führen; die be\%,ïglich der Präcision wenig vortheilhaft gewähIten Worte „Eindruck der Weichtheile“ kann mit Zuhilfenahme der beigegebenen Figur vielleicht einigermaassen genügend ergänzt werden.

In den von mir beschriebenen und abgebildeten Fïllen sind die Myocommata nicht substantiell erhalten, sondern treten nur als mehr weniger feine Furchen auf, worauf wir unsere Ansicht gründeten, dass Sehnensubstanz überhaupt nicht zur Verkalkung gelangen könne ${ }^{2}$ ). Dieser Punkt bildet eine der wichtigsten Grundlagen der Theorie der Erklärung der Structurerhaltung der Muskelfasern in der Petrificationsmasse iberhaupt, in der ich bis jetzt keinen Widerspruch erfahren habe.

In einem etwaigen Nachweis der Sehnensepten der Rumpfmuskeln bei Pleurosaurus läge aber ein Widerspruch, der von unserer Seite zu lösen wäre; die von W. Pütz gezeichnete Abbildung zeigt nun die segmentalen Querstreifen, welche „Eindricke" der Myocommata sein sollen, innerhalb des deutlichen Kïrpersaumes bei der allgemeinen Beleuchtung der Erhabenheiten der Platte von rechts, als einen nach aussen sich verlierenden dunkeln Strich, welcher von oben her durch eine Lichtlinie erhöht ist; der zeichnerische Eindruck ist der, dass man hier nicht

1) Ich mache nachträglich noch auf die schöne Abbildung bei N e w b erry (Palaeoz. fish. of North.-Am., Un. St. Geol. Surv., Monographs XVI, Pl. XLIX, Fig. 2, tail and posterior body showing myocommata or septa dividing them) aufmerksam.

2) Mikroskopische Querschnittsbilder, welche hie und da an Querschnitte von Sehnenfasern erinnern, erwiesen sich in allen Fällen als posthum oder vor der Verkalkung veränderte und geschrumpfte Partieen von beiderseits oder ringsum in normalerer Struktur fortsetzenden Muskelfasern; ich betone dies als das Resultat verschiedentlich rorgenommener Nachprüfungen. 
eine Furche, sondern eine erhöhte Partie vor sich habe. Ist dieser Eindruck correkt, so wird man diesen erhöhten Streifen nur der Gesteinsmasse selbst zuschreiben können und seine Entstehung auf die Ausfüllung der Myocommata-Furchen zwischen der verkalkten Muskulatur zurückführen müssen, wie dies bei Fischen oft $\mathrm{zu}$ beobachten and von mir erwähnt ist. Die verkalkte Muskulatur erwähnt nun W. D a m e s an demselben Exemplar an mehreren Stellen des Knochengerüstes selbst; hier scheint sie sich zwischen den Knochen festgehalten zu haben, während sie sich ausserhalb desselben (d. h. im Bereich der „MyocommataEindriicke") wohl bei der Auseinanderspaltung der Platten abgesplittert oder vorher chemisch anfgelöst haben kann.

Ist diese Deutung nicht richtig und sind die Striche, welche die Myocommata andeuten sollen, wirkliche $\mathrm{V}$ erti ef u $\mathrm{n} g$ e $\mathbf{n}$ in der Gesteinsmasse (was die Abbildung nicht zeigt), so muss nach einer anderen Deutung gesucht werden. Von einem einfachen "Abdruck" der Myocommata in weichem Zustande kann natürlich bei einem Reptil nicht die Rede sein, welches ganz zweifellos nach dem Lrtheile Aller, wie sein lebender naher Verwandter S phenod on, eine mit Hornschuppen versehene Haut batte; besonders in dem vorliegenden Falle, dass diese Hornsehuppen nicht auch im Gesteine abgedrïclst sind, kann von einem "Abdruck" der weichen Myocommata nicht die Rede sein. Wir müssten also hier einen vorhergegangenen Verkalkungsprozess des Selınengewebes annehmen. Fïr diesen Fall möchte ich daran erinnern, dass ich bei Fiscben auch eine unter gewissen Umständen stattfindende Verkalkung der dem Sehnengewebe histologisch viel näher stehenden Cutis nachgewiesen habe, welche ich anf abnorme Umstände des Hautgewebes zur Zeit der Laichperioden zurückführte (vgl. Arch. für mikrosk. Anatomie Bd. XXXXIV, S. 111). Meine Uebertragung dieser Ansicht auf die von Eb. Fra as beobachtete Kammbildung bei Ichthyosaurus hat, wie ich mir nach brieflicher Mittheilung zu erwähnen erlaube, die lebhafte Zustimmung bei Herrn Prof. Fr a a s gefunden. Es wäre möglich, dass anch hier im Princip Aehnliches vorläge; von jener sexuell hervorgerufenen Erweichung und Schwellung der Cutis könnten auch besonders in der dorsalen Thorakalregion Verbindungsstellen der Cutis nit den Myocommata, ja diese selbst in einiger Tiefe ergriffen werden und demgemëss auch verkalkt sein. Diese $\mathbf{M} \ddot{o} \mathrm{~g}$ - 
lichkeit ist also vorhanden, wenn mir auch unsere erstere Deutung die wahrscheinlichere ist, dass einfach auch hier, wie ïberall, ein von der Gesteinsmasse gebildetes Negativ der Myocommatafurchen zwischen den verkalkten Myomerstreifen der Rumpfmuskulatur vorliege.

Dass wir hierauf so eingehend zurückgekommen sind, geschah besonders in Anregung zu etwas genaueren Darstellungen derartiger Befunde unter ausführlicherer Berücksichtigung der grundlegenden Arbeiten, damit ein Fortschritt in dieser Sache überhaupt möglich sei. Die Ergründung dieser Erscheinung ist ja nicht etwa eine aussichtslose nebensächliche Spielerei paläiohistologischer Untersuchungen; sie hat nicht nur - wie der Nachweis der Identität der Erscheinung mit den älteren $H$ a r t in $g$ 'schen Petrifications-Experimenten an weichen organischen Substanzen darlegt, eine allgemeine Bedeutung, sondern sie kann auch besonders paläontologisch vielfach verwendet werden; ich habe selbst eine Anzahl kleinerer derartiger Beispiele schon früher besprochen und velweise heute nur noch auf die Bemerkung, welche Rauff bezüglich der Erhaltung fossiler Kalkschwämme macht (vgl. Neues Jahrb. für Mineralogie 1895 und Sitzungsber. der niederrh. Ges. in Bonn (natw. Section) 1894 S. 100-113) und auf meine Anwendung der Schrumpfungserscheinungen bei der eintretenden Verkalkung bezïglich der Erklärung eigenartiger Strukturen der erhärteten Hornfasern bei Acanthodes ( $\mathrm{S} \mathrm{chwalbe's:} \mathrm{Morphol.}$ Arbeiten Bd. VI, S. 200).

Ein weiterer Punkt neuerer Untersuchung betrifft das Auftreten der Muskelverkalkung bei.Pycnodontiden; ich selbst habe früher keinen Fall davon beobachtet and dies darauf zurückzufihren gesucht, dass dieselben keine Vertebratenfresser seien und eine in ihren Gedärmen gelöste Menge von Knochensubstanz sogleich nach dem Absterben noch den Process der Phosphoritisirung nicht rasch genug einleiten konnte, welche Anfangsmenge in Fällen eintretender völliger Phosphoritisirung dann durch allmähliche Zuführung weiterer Mengen löslicher phosphorsaurer Salze von aussen her vervollstaindigt werden musste.

Das paläontologische Museum in München hat nun in nenerer Zeit ein prachtrolles Exemplar des Pycnodontiden Mesturus aus dem lithogr. Schiefer erworben, welches die phosphoritisirte 
Muskulatur an Stellen des abgebrochenen Schuppenkleids schon makroskopisch in deutlicher Weise zeigt; die Dicke der Lage ist gar nicht einmal gering und Herr Dr. Plieninger verfertigte Dünnschliffe, welche nicht nur die Muskelstructur in deutlichster Weise zeigten, sondern auch die bei Ischyodus, Caturus and L e p t o th e u t is beobachteten mikroskopischen Krystallchen von Kalkspath in ganz ähnlicher Einlagerung, Erhaltung und Grössenentwickelung aufweisen, was selbst in seiner Nebensächlichkeit die grosse Constanz der A rt des Phosphoritisirungsprocesses beweist.

Wenn nun clies die Bedeutung der Pycnodontiden im oben angeführten Sinne erschüttern könnte, so ist dagegen zu bemerken, dass gerade Mesturus von dem Allgemeintypus der Pyenodontiden abweicht und seine Bezahnung auch auf die Möglichkeit ausgedehnterer Vertebratennahrung hinweist. Ueberhaupt ist wohl die Molluskennahrung bei den Pycnodontiden ebensowenig exclusiv, wie bei den Holocephalen, wie es auch bei der Ernährungsweise nicht blos auf die Art der Bezahnung allein, sondern auf die Entwickelung von Körperform und Flossenskelet, d. h. die Bewegungsart ankommt, da Vertebratenfresser auch viel bewcglichere Geschöpfe sein müssen; anch hierin ist M e sturus nach der Widerstandsfähigkeit und Festigkeit der Flossenstrahlen, sowie der Entfaltung eines Schwanzstieles unter den Pycnodontiden bevorzugt zu nennen. Auch gewebig ist bei ibm das Haut- und Knochenskelet vollständiger als bei den tibrigen Pycnodontiden; während z. B. in den Schuppen das Dentin bei gewissen Arten von Gyrodus nur sehr spärlich oder anch fehlend zu beobachten ist, besteht die reiche Dermalskulptur bei Mesturus gänzlich ans Dentin ${ }^{1}$ ); dem Auftreten dieses älteren Strueturtypus gemäss ist

1) Ich meine hiermit wirkliches Dentin, uicht die von Skupin sogenannten und fälschlich zum Dentin gerechneten Osteinröhrchen; Skup in hat auch das vereinzelte Auftreten von echtem Dentin bei Gyrodus nicht beobachtet (Arch. f. Naturgesch. Jahrg. 1896. Bd. I, Heft 2), wie er auch Mesturus nicht kennt; er verallgemeinert trotzdem seine isolirten Beobachtungen auf die Pycnodontiden, hier wie in anderen Fällen. Es ist zu bedauern, dass in dieser jedenfalls Heissigen Arbeit wichtige, zur Discussion gestellte Fragen histiogenetischer Entwicklung des gesammten Hautskelets (vgl. Jahrb. f. Miner. etc. 1895 , S. 165) durch die dabei beliebte Begriffsaufstellung einfach ignorirt worden sind; wir kommen sehr bald eingehender hierauf $\mathrm{zu}^{-}$ 
der Bestand des gesammten Hautskelets vollständiger als bei Gyrodus, ebenso das Knochenwachsthum normaler und ohne alle Degenerationsanzeichen; mir scheint auch dies für eine vorwiegend normalere Ernährungsweise zu sprechen.

Was den Nachweis von Hodenpetrificirung bei Eurycormus betrifft, so erwähnt A. S. Woodward, Cat. of foss. fish. Brit. Museum III S. 353, offenbar ohne noch meine auch 1895 erschienene Abhandlung zu kennen, von einem E ary cormus speciosus ans Kehlheim: "apparently showing traces of the ovaries."

(Aus der Anatomischen Anstalt Freiburg i. B.).

\title{
Beiträge zur Lehre von den Sinnesorganen der Hatteria punctata.
}

\author{
Von \\ Gakntaro Osawa aus Japan.
}

Hierzu Tafel XVI, XVII u. XVIII und 22 Textfiguren.

In meinen wiederholten Publicationen habe ich bis jetzt über das Skelet-, Muskel- und Nervensystem, ferner über alle Eingeweideorgane der männlichen wie der weiblichen $\mathrm{H}$ a t t e ria sowie über die Hant dieses Thieres berichtet ${ }^{1}$ ). In der vorliegenden Arbeit möchte ich nın zum Schlusse noch die Verhältnisse der Sinnesorgane, wie des Gesichts-, Geruchs- and Sehorgans des betreffenden Thieres zur Rede bringen, um so der ganzen Monographie über die $\mathrm{Hatteria}$ eine vollständige

rück, da uns diese "vergleichenden Studien" leider nicht weiter gebracht haben.

1) Osawa, Beitrag zur feineren Struktur des Integumentes der Hatteria punctata. Dies Arch. Bd. 47. 1896. - Derselbe, Beiträge zur Lehre von den Eingeweiden der H. p. Dies Arch. Bd. 49. 1897.Derselbe, Beiträge zur Anatomie der H. p. Dies Arch. Bd. 51. 1898. - Derselbe, Nachtrag zur Lehre von den Eingeweiden der H. p. Dies Arch. Bd. 51. 1898. 